\title{
X-ray diffraction gratings: Precise control of ultra-low blaze angle via anisotropic wet etching
}

Dmitriy L. Voronov, Paul Lum, Patrick Naulleau, Eric M. Gullikson, Alexei V. Fedorov, Howard

A. Padmore

\begin{abstract}
Diffraction gratings are used from micron to nanometer wavelengths as dispersing elements in optical instruments. At shorter wavelengths, crystals can be used as diffracting elements, but due to the 3D nature of the interaction with light are wavelength selective rather than wavelength dispersing. There is an urgent need to extend grating technology into the $x-$ ray domain of wavelengths from 1 to $0.1 \mathrm{~nm}$, but this requires the use of gratings that have a faceted surface in which the facet angles are very small, typically less than $1^{\circ}$. Small facet angles are also required in the extreme ultra-violet and soft x-ray energy ranges in free electron laser applications, in order to reduce power density below a critical damage threshold. In this work, we demonstrate a technique based on anisotropic etching of silicon designed to produce very small angle facets with a high degree of perfection.

X-ray diffraction gratings operate at very shallow incidence angles due to critical angle constraints. For the same reason, facetted or blazed gratings should have a very small blaze angle, ranging from a few degrees for EUV and soft x-ray spectrometers down to a few tenths of a degree for hard $\mathrm{x}$-rayapplications such as X-ray free electron lasers. Blazed $\mathrm{x}$-ray gratings are produced by diamond ruling or fabricated using holography and ion beam etching method. These classical techniques are capable of providing high quality gratings with relatively deep grooves and high and medium blaze angles. However, fabrication of triangular grooves with small blaze angles (below $5^{\circ}$ ) is very challenging for both techniques. To reduce the blaze angle of the ruled gratings, an additional technological step of ion sputtering or/and plasma etch is required. This technique is based on etching of a silicon substrate through a patterned gold layer and relies on the different sputter/etch rates of gold and silicon which result in significant reduction of the relief of the grooves transferred into the $\mathrm{Si}$ substrate. The metal/silicon etch ratio depends on plasma parameters which should be carefully optimized to achieve the required blaze angle.
\end{abstract}

Although successful implementation of the angle reduction approach has been reported, the overall ruling/etch process is rather complex and expensive which results in high cost and production time of the gratings. In this work, we suggest a simple and effective way to make ultra-low blaze angle gratings for EUV, soft and hard x-rays by anisotropic wet etch of Si single crystals. The etch rate of a Si surface in alkali solutions $\left(\mathrm{NaOH}, \mathrm{KOH}\right.$, and $\left.\mathrm{NH}_{4} \mathrm{~F}\right)$ is highly dependent on the crystallographic orientation of the surface. Since (111) planes have the lowest etch rate, an etched Si surface tends to be faceted with (111) terraces which can be used for making V-shaped grooves. This method was originally suggested for production of echelle gratings for optical-fiber communications systems and adopted later for making 
multilayer blazed gratings. In this work, we investigate the wet etching process in terms of the possibility to make very shallow grooves with extreme blaze angles. We will demonstrate that the approach is very promising for grazing incidence $x$-ray gratings since it offers the unique capability of precise control of the blaze angle during the fabrication process.

The facet slope of anisotropically etched blazed gratings is defined mostly by the orientation of the (111) planes underlying the etched surface. However, a detailed analysis of the groove shape evolution in the course of etching shows that there is some deviation of the facet surface from the exact (111) orientation. The facet starts out as a vicinal surface and then slowly approaches the (111) surface. The evolution of the facet shape of a 10- $\mu \mathrm{m}$ period grating with a nominal blaze angle of $6^{\circ}$ is illustrated in Fig. 1 where AFM images of a groove at different stages of the etch process are shown. The surface of asymmetrically cut Si single crystals is patterned with silicon nitride stripes which serve as a hard mask (Fig. 1(a)) during wet etching. The etching of a Si surface occurs through a step-flow mechanism by formation of atomic pits and motion of atomic steps which are dominant etch species. Since the $6^{\circ}$ miscut surface has a lot of atomic steps, it is attacked by $\mathrm{KOH}$ much more vigorously than the (111) planes. The etching front goes down relatively fast and slows down significantly as it reaches a (111) plane, resulting in the formation of a blazed facet. In the middle of the etch process, the groove consists of a slanted part which has an orientation close to (111) planes and a horizontal part which is the miscut surface (Fig. 1(b)). There is a gradual transition between the two parts with a continuous change of the surface angle from $6^{\circ}$ to $0^{\circ}$.

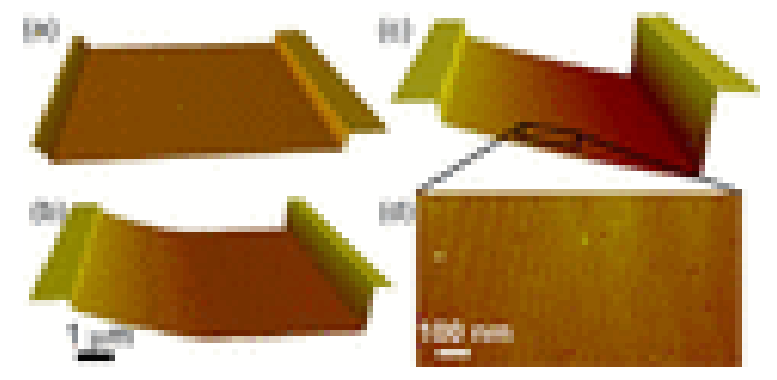

FIG. 1. Morphology of a groove of a blazed grating with period of $10 \mu \mathrm{m}$ period and the nominal blaze angle of $6^{\circ}$ : before wet etching (a), after $30 \mathrm{~min}$ (b), and $3 \mathrm{~h}$ (c) of etch in $\mathrm{KOH}$, after additional $\mathrm{NH}_{4} \mathrm{~F}$ etch (d).

Eventually the miscut surface collapses and the grooves are fully developed (Fig. 1(c)). However, the gradual change of the slope angle is inherited by the blazed facets resulting in their curvature and variation of their tilt from $6^{\circ}$ in the upper part to $5^{\circ}$ in the bottom part. A closer look on the facet surface morphology shows that the facet is not exactly (111) plane. The surface morphology after additional short etching in ammonium fluoride $\left(\mathrm{NH}_{4} \mathrm{~F}\right)$, which reveals the fine atomic structure of the surface, is shown in Fig. 1(d). The facet surface consists of (111) terraces and atomic steps of the same sign, meaning that the facet surface is not exactly (111) plane but a vicinal surface with a residual miscut. Moreover, the atomic step density is not uniform and increases from the upper part of the facets to the bottom part of the facets. The nonuniformity in step density inherited from the previous etch stage is consistent with the concave shape of the facets.

As soon as blazed facets are fully developed the etch process slows down significantly but does not stop completely. Since the etch rate is proportional to the step density and the step density 
is not uniform over the facet length, the upper and bottom parts of the facets are etched with different etch rates. The residual etch rate anisotropy causes further evolution in surface morphology and shape of the facets. The step density gradually reduces and becomes more uniform which results in flattening of the facets and approaching of their average tilt to the original miscut angle of the Si single crystal.

One can speculate that the grating facets should have some non-zero curvature even after very long etching due to an edge effect caused by the fact that a particular groove is confined by the mask stripes. Let us assume that original groove surface (Fig. 1(a)) has zero miscut, i.e., is an exact (111) plane. According to the step flow etch mechanism, the net etch rate of a (111) surface at any particular point of the surface is defined by the number of steps passing this point per unit time. In the central part of a facet, the steps come from both the right and the left sides, while in the vicinity of the mask stripes (at the distance comparable to the natural width of (111) terraces), the steps can come only from one side, so the etch rate here should be lower. The lower etch rate results in slight bending of the very edges of the facets, making them vicinal surfaces with an increased number of steps (of the same sign). Eventually, the total step density at the edges becomes the same as in the center of a facet and a non-zero steady-state curvature is established. This model should result in atomic steps of opposite sign in the upper and bottom parts of the tilted facets. However, this has never been observed in our experiments, probably because edge effects are localized in the areas next to the mask stripes and not accessible due to the finite width of the AFM probe. More realistically, the edge effects are very weak, and their contribution to the total facet curvature is negligible.

Slow changes in the facet shape during extended $\mathrm{KOH}$ etch allows precise control of the slope of the facets and hence the blaze angle of the blazed grating through control of the etching time. The etching should proceed until a desirable angle value is achieved and then stop. In the following, we will demonstrate fabrication of x-ray gratings with ultra-low blaze angles of $0.65^{\circ}$ and $0.4^{\circ}$.

The fabrication approach described above was applied to fabrication of a 200 lines $/ \mathrm{mm}$ variable line spacing (VLS) grating for the Extreme Ultra-Violet Lithography (EUVL) beamline BL12.0 at the Advanced Light Source (ALS). Parameters of the grating are dictated by the monochromator design which operates at a constant deviation angle of $166^{\circ}$ and the requirement for providing the highest possible throughput at a wavelength of $13.5 \mathrm{~nm}$. The optimal value of $0.65^{\circ}$ of the blaze angle which provides diffraction efficiency up to $76 \%$ in the 1 st diffraction order was found by simulation of the diffraction efficiency using PCGrate 6.1 electromagnetic code. The precision of the blaze angle is of great importance since a deviation as small as $\pm 0.2^{\circ}$ from the optimal value would result in an almost two-fold reduction of the blazed order efficiency and increase of efficiency of the zero or second diffraction orders (Fig. 2). The non-optimal blaze angle and groove profile (Fig. 3(a)) were the main reasons for the low efficiency of $23 \%$ of the commercial grating originally installed in the monochromator. 


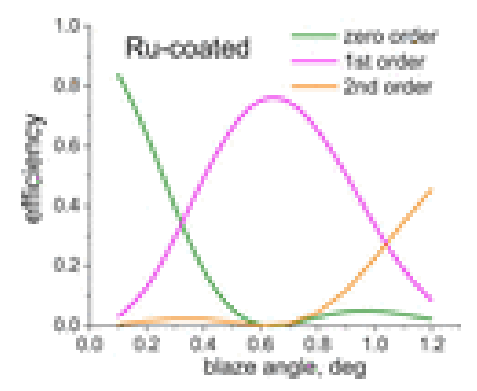

FIG. 2.Dependence of theoretical absolute efficiency on blaze angle for zero, 1st, and 2nd diffraction orders of a Rucoated 200 lines $/ \mathrm{mm}$ diffraction grating at a wavelength of $13.5 \mathrm{~nm}$ and an incidence angle of $82.365^{\circ}$.

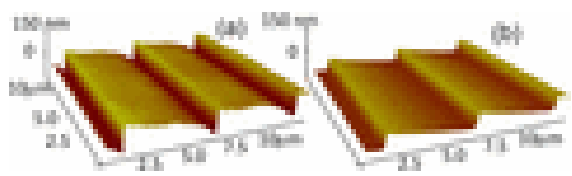

FIG. 3.AFM images of the commercial diamond ruled grating (a) and the anisotropically etched grating \#1 with groove density of 200 lines $/ \mathrm{mm}$ and an average blaze angle of $0.75^{\circ}$ (b).

The single crystal Si substrate with a miscut angle of $1^{\circ}$, thickness of $5 \mathrm{~mm}$, and diameter of $100 \mathrm{~mm}$ was oxidized to create a $100 \mathrm{~nm}$ thick silicon oxide layer and then spin-coated by AZ BARLi II antireflective coating and PFI88A7 photoresist. The VLS pattern was recorded on a resist coated Si substrate by direct write lithography using a Heidelberg DWL66 laser writer. The pattern was then transferred to the silicon oxide layer by reactive ion etch using oxygen and then $\mathrm{CHF}_{3}$ plasma. Anisotropic wet etch in $20 \% \mathrm{~m} / \mathrm{m} \mathrm{KOH}$ aqueous solution was performed at room temperature. The groove profiles of the grating measured with AFM before wet etch and after 2 and $3.5 \mathrm{~h}$ are shown in Fig. 4. After $2 \mathrm{~h}$ of etching, the angle of the tilt of the facet varied from $0.92^{\circ}$ to $0.07^{\circ}$ across the facet length and the average slope was about $0.45^{\circ}$. The AFM data were used to compose a virtual profile of a blazed grating assuming an antiblaze angle of $5^{\circ}$ (green curve in Fig. 5(a)). Dependence of diffraction efficiency of the virtual grating on wavelength was calculated for the constant incidence angle of $82.365^{\circ}$, which corresponds to the BL12.0 monochromator geometry. Since the blaze angle of the grating is not optimal, the maximum of the efficiency is shifted towards shorter wavelengths from the target value of $13.5 \mathrm{~nm}$ (Fig 5(b)).

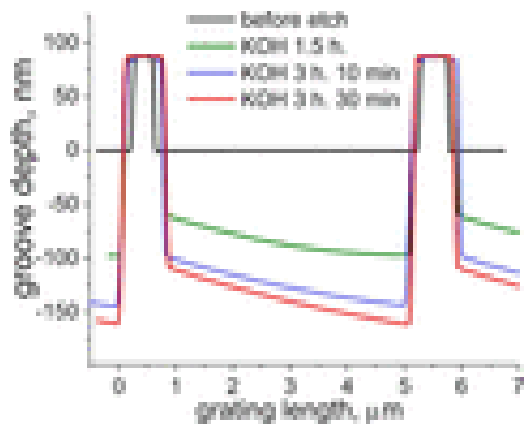

FIG. 4.Average groove profile of the 200 lines $/ \mathrm{mm}$ grating with a miscut angle of $1^{\circ}$ before wet etching, and after etching in $20 \% \mathrm{KOH}$ solution at room temperature for $1.5 \mathrm{~h}, 3 \mathrm{~h} 10 \mathrm{~min}$, and $3 \mathrm{~h} 30 \mathrm{~min}$. 


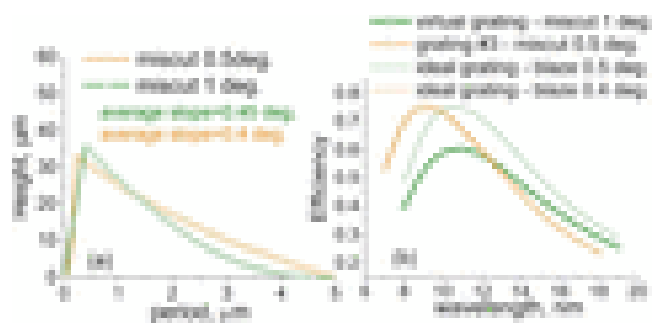

FIG. 5.Groove profile (a) and calculated diffraction efficiency (b) of the gratingsobtained by etch of Si single crystals with miscut angles of $1^{\circ}$ and $0.5^{\circ}$ (curves with circles and triangles, respectively). The efficiency of an ideal grating with plane facets and blaze angles of $0.4^{\circ}$ and $0.5^{\circ}$ is shown by the thin curves.

The same grating (Grating \#1) was etched for another $1.5 \mathrm{~h}$, and so the total etch time was 3.5 $\mathrm{h}$. The AFM measurements showed that the slope of the facet is somewhat larger than the desired one (red curve in Fig. 6(a)). Another grating (Grating \#2) was etched for a shorter time of $3 \mathrm{~h} 10 \mathrm{~min}$ (blue curve in Fig. 6(a)). The etching was stopped when the average slope angle measured approached the optimal value. Then, the oxide mask was removed by HF for both the gratings, and isotropic etchwith RCA-1 solution (mixture of ammonium hydroxide and hydrogen peroxide) was performed to remove the silicon nubs left by the $\mathrm{KOH}$ step to finalize the saw-tooth shape of the grooves (Fig. 3(b)). The gratings \#1 and \#2 have slightly concave blazed facets and average blaze angles of $0.75^{\circ}$ and $0.63^{\circ}$, respectively (red and blue curves in Fig. 6(a)).

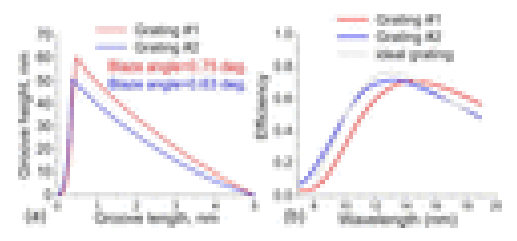

FIG. 6.Average groove profile (a) and measured diffraction efficiency (b) of the Grating \#1 (red curve) and grating \#2 (blue curve). Theoretical efficiency of an ideal grating with perfectly plane facets is shown with a grey curve.

The diffraction efficiency of the gratings coated with a Ru coating was measured at the $x$ ray optics testing beamline BL6.3.2 at the ALS. Grating \#1 has a very high efficiency of $69.6 \%$ at the design wavelength of $13.5 \mathrm{~nm}$ with the maximum of the efficiency curve very close to the desired wavelength. The maximum of efficiency of grating \#2 matches $13.5 \mathrm{~nm}$ perfectly due to an optimal blaze angle of $0.63^{\circ}$. Measured efficiency of $71.8 \%$ of grating \# 2 is almost as high as the theoretical efficiency calculated for an ideal grating with triangular grooves and perfectly flat facets (grey curve in Fig. 6(b)). This indicates that the residual curvature of the blazed facet of the anisotropicallyetched gratings does not significantly affect efficiency.

The impact of facet curvature on grating efficiency should be considered for specific arrangements of the grating and diffraction geometry. The geometry defines the portion of the facet area which is illuminated (a part of the facet can be self-shadowed) and an effective blaze angle can be estimated as an average slope of the non-shadowed part for the curved facet. From general considerations, the impact of the curvature can be significant when deviation of the facet surface from a plane is comparable to the groove depth, which is a quantity which defines a phase shift of $\lambda / 2$ for the adjacent grooves of the grating. For example, the curvature of the virtual grating (green curve in Fig. 5(a)) is rather substantial and results in a sag as large 
as $11 \mathrm{~nm}$ which is almost $1 / 3$ of the groove depth of $36 \mathrm{~nm}$. The calculated diffraction efficiency of the grating is substantially lower than the one for an ideal grating with ideally flat facets (thin green curve in Fig. 5(b)). To make a high efficiency grating with very low blaze angles, it is better to start with a Si single crystal with a lower miscut angle. Grating \#3 was fabricated from a $0.5^{\circ}$ miscut Si crystal using the $\mathrm{KOH}$ process described above. The final profile of the grating is shown in Fig. 5(a) by the orange curve. The grooves of the gratings have much flatter facets, and the sag does not exceed $4 \mathrm{~nm}$ which is almost of an order of magnitude less than the groove depth. The efficiency of grating \#3 was calculated for the same geometry as for the virtual grating. The efficiency is much higher than for the virtual grating and close to the efficiency of the ideal grating with perfectly flat facets (orange curve in Fig. 5(b)) demonstrating a negligible effect of the residual facet curvature of grating \#3.

In summary, the kinetics of the anisotropic wet etching for grating grooves leads to curved blazed facets composed of (111) terraces and atomic steps with non-uniform distribution of the step density across the facet length. Extended etching results in a slow change of the facet curvature and the average slope angle. This provides way for precise control of the blaze angle of x-ray gratings which is particularly important for grazing incidence x-ray gratings with ultra-low blaze angles. The approach was used for fabrication of a 200 lines $/ \mathrm{mm}$ VLS grating with blaze angle of $0.65^{\circ}$ for EUV beamline BL12.0 at the ALS. Due to the almost perfect groove profile, the efficiency of the anisotropically etched gratings is close to the theoretical value and exceeds the efficiency of the standard ruled commercial grating originally installed in the beamline monochromator more than by a factor of 3. Such exquisite control of the facet angle for low blaze angles is important for a wide range of applications from EUV lithography to ultra-high power soft x-rays FELs, to extension of reflection grating performance into the multi-keV $x$ ray region.

Advanced Light Source and Molecular Foundry are supported by the Director, Office of Science, Office of Basic Energy Sciences, of the U.S. Department of Energy under Contract No. DEAC02-05CH11231. 